\title{
Novel phase transition in charged dilaton black holes
}

\author{
Amin Dehyadegari, ${ }^{1}$ Ahmad Sheykhi, ${ }^{1,2, *}$ and Afshin Montakhab ${ }^{1, \dagger}$ \\ ${ }^{1}$ Physics Department and Biruni Observatory, Shiraz University, Shiraz 71454, Iran \\ ${ }^{2}$ Research Institute for Astronomy and Astrophysics of Maragha (RIAAM), P.O. Box 55134-441, Maragha, Iran
}

\begin{abstract}
We disclose a novel phase transition in black hole physics by investigating thermodynamics of charged dilaton black holes in an extended phase space where the charge of the black hole is regarded as a fixed quantity. Along with the usual critical (second-order) as well as the first-order phase transitions in charged black holes, we find that a finite jump in Gibbs free energy is generated by dilaton-electromagnetic coupling constant, $\alpha$, for a certain range of pressure. This novel behavior indicates a small/large black hole zeroth-order phase transition in which the response functions of black holes thermodynamics diverge e.g. isothermal compressibility. Such zeroth-order transition separates the usual critical point and the standard first-order transition curve. We show that increasing the dilaton parameter $(\alpha)$ increases the zeroth-order portion of the transition curve. Additionally, we find that the second-order (critical) phase transition exponents are unaffected by the dilaton parameter, however, the condition of positive critical temperature puts an upper bound on the dilaton parameter $(\alpha<1)$.

PACS numbers: 04.70.-s, 05.70.Ce, 04.70.Dy, 04.60.-m
\end{abstract}

\section{INTRODUCTION}

Since the discovery of black holes thermodynamics in 1970's by Bekenstein and Hawking [1, 2], physicists have been speculating that there should be some kind of thermodynamic phase transition in this gravitational system. Hawking and Page were the first who discovered a first order phase transition of thermal radiation-large black holes in the background of Schwarzschild anti-de-Sitter (AdS) spacetime [3]. Later, by considering charged black holes, a phase transition was shown to occur between small-large black holes [4]. Such a phase transition has been associated to a liquid-gas transition such as the one occurring in the Van der Waals system [6, 28]. Recently, phase transition of charged AdS black holes has attracted much attentions. It was shown that thermodynamic properties of charged AdS black holes admit a first order phase transition between large and small black holes occurs which is analogous to the Van der Waals liquid-gas phase transition $[7,8]$. In this perspective, thermodynamic analysis are improved in an extended phase space in which the cosmological constant and its conjugate variable are considered as thermodynamic pressure and volume, respectively. It has been demonstrated that the first law of black hole thermodynamics is consistent with the Smarr relation provided the mass of black hole is identified as the enthalpy [9]. Phase transition and critical behaviour of black holes in an extended phase space have been investigated in ample details (see [10-18] and references therein). In all these works ([7-18]) the cosmological constant (pressure) is considered as a variable quantity and the charge of the black holes is fixed.

In another approach towards thermodynamic phase

\footnotetext{
*Electronic address: ashykhi@shirazu.ac.ir

${ }^{\dagger}$ Electronic address: montakhab@shirazu.ac.ir
}

space of black holes, it was shown that one can think of variation of charge $Q$ of a black hole and keep the cosmological constant as a fixed parameter. The motivation for this assumption comes from the fact that the charge of a black hole is a natural external variable which can vary [19]. Besides, the cosmological constant is related to the background of AdS geometry and it is more natural to take it as a constant, rather than a variable quantity [19]. This alternative view of such a phase space and more physically conventional description of the phase transition naturally leads to a meaningful response function and a more accurate analogy with the Van der Waals fluid [19]. Indeed, in this perspective, the critical behavior occurs in $Q^{2}-\Psi$ plane, where $\Psi=1 / 2 r_{+}$is the conjugate of $Q^{2}$ [19]. It was shown that a small-large black hole phase transition occurs with an associated critical point $\left(T_{c}, Q_{c}^{2}, \Psi_{c}\right)$ with complete analogy with the Van der Waals fluid system [19].

A discontinuity in the derivatives of Gibbs free energy with respect to temperature characterizes the type of phase transition that occurs in thermodynamic system. A first-order phase transition has a discontinuity in the first derivative which is entropy, i.e. $(\partial G / \partial T)=S$, and second-order (critical) phase transition has discontinuity (singularity) of $\left(\partial^{2} G / \partial^{2} T\right)$. Therefore, a lesserknown zeroth-order phase transition has a discontinuity in the Gibbs free energy itself, which was discovered in superfluidity and superconductivity [20]. The author of Ref.[20] also showed that a zeroth-order phase transition occurs in Bogolyubov's model of a weakly nonideal Bose gas. On the other hand, a novel reentrant phase transition has been recently observed to accompany the standard first-order phase transitions in black holes[21-25]. Motivated by the above novel phase transitions, we intend to present a study of small-large phase transitions in charged dilaton black holes. Therefore, we analyze the possible phase transitions in extended phase space for fixed charge where the space-time geometry is described 
by Einstein-Maxwell-dilaton gravity [26]. We find that the presence of dilaton parameter leads to a region of the phase diagram which allows for zeroth, first and secondorder phase transition where the zeroth-order separates the first and second order transition, along the transition curve. The extension of such a region becomes larger with increasing dilaton parameter.

This Communication is structured as follows: in section II, we study thermodynamics of $(d+1)$-dimensional charged dilaton black holes in the presence of Liouvilletype dilaton potential. In section III, we investigate critical behavior of dilaton black holes. In section IV, we study equation of state of charged dilaton black holes. The last section is devoted to summary and conclusions.

\section{BASIC THERMODYNAMICS OF CHARGED DILATON BLACK HOLE}

The action of $(d+1)$-dimensional spacetime in Einstein-Maxwell theory with a scalar dilaton field $(\varphi)$, reads [27]

$$
\begin{aligned}
\mathcal{I}= & \frac{1}{16 \pi} \int d^{d+1} x \sqrt{-g}\left(\mathcal{R}-\frac{4}{d-1}(\nabla \varphi)^{2}-\mathcal{V}(\varphi)\right. \\
& \left.-e^{-4 \alpha \varphi /(d-1)} F_{\mu \nu} F^{\mu \nu}\right)
\end{aligned}
$$

where $F_{\mu \nu}=\partial_{[\mu} A_{\nu]}, A_{\nu}$ is the vector potential and $\alpha$ is the coupling parameter of dilaton with Maxwell field. Hereon, $\mathcal{V}(\varphi)$ is the dilaton potential which has the following form $[26,27]$

$$
\mathcal{V}(\varphi)=2 \Lambda e^{4 \alpha \varphi /(d-1)}+\frac{(d-1)(d-2) \alpha^{2}}{b^{2}\left(\alpha^{2}-1\right)} e^{4 \varphi /[(d-1) \alpha]}
$$

where $b$ is a positive arbitrary constant. In the absence of dialton field $(\alpha=0)$ the above potential reduces to $\mathcal{V}(\varphi) \rightarrow 2 \Lambda$, and thus one may interpret $\Lambda$ as the cosmological constant. The $(d+1)$-dimensional spherical symmetric metric is given by

$$
d s^{2}=-f(r) d t^{2}+\frac{d r^{2}}{f(r)}+r^{2} R(r)^{2} d \Omega_{d-1}^{2},
$$

where $d \Omega_{d-1}^{2}$ is the line element of an unit $(d-1)$ sphere with the volume $\omega_{d-1}$. Applying ansatz $R(r)=$ $e^{2 \alpha \varphi /(d-1)}$, one can show that [26]

$$
\begin{array}{r}
f(r)=\frac{2 \Lambda\left(\alpha^{2}+1\right)^{2} r^{2(1-\gamma)}}{(d-1)\left(\alpha^{2}-d\right) b^{-2 \gamma}}-\frac{(d-2)\left(\alpha^{2}+1\right)^{2} b^{-2 \gamma} r^{2 \gamma}}{\left(\alpha^{2}-1\right)\left(\alpha^{2}+d-2\right)} \\
-\frac{m}{r^{(d-1)(1-\gamma)-1}}+\frac{2 q^{2}\left(\alpha^{2}+1\right)^{2} r^{2(d-2)(\gamma-1)}}{(d-1)\left(\alpha^{2}+d-2\right) b^{2 \gamma(d-2)}},(4) \\
\varphi(r)=\frac{(d-1) \alpha}{2\left(1+\alpha^{2}\right)} \ln \left(\frac{b}{r}\right), \quad A_{t}=\frac{q b^{\gamma(3-d)}}{\Pi r^{\Pi}},
\end{array}
$$

where $\gamma=\alpha^{2} /\left(\alpha^{2}+1\right), \Pi=(d-3)(1-\gamma)+1, b$ is a positive arbitrary constant, $m$ and $q$, respectively, are related to the total mass and electric charge of the black hole [26]

$$
M=\frac{b^{\gamma(d-1)}(d-1) \omega_{d-1}}{16 \pi\left(\alpha^{2}+1\right)} m, \quad Q=\frac{q \omega_{d-1}}{4 \pi} .
$$

Inasmuch as the event horizon is defined by the largest root of $f\left(r_{+}\right)=0$, one can write $m$ in terms of $r_{+}$. Temperature, entropy and electric potential of EinsteinMaxwell-dilaton black holes are obtained as [26]

$$
\begin{gathered}
T=-\frac{(d-2)\left(\alpha^{2}+1\right) b^{-2 \gamma}}{4 \pi\left(\alpha^{2}-1\right)} r_{+}^{2 \gamma-1}-\frac{\Lambda\left(\alpha^{2}+1\right) b^{2 \gamma}}{2 \pi(d-1)} r_{+}^{1-2 \gamma} \\
-\frac{q^{2}\left(\alpha^{2}+1\right) b^{-2 \gamma(d-2)}}{2 \pi(d-1)} r_{+}^{(2 d-3)(\gamma-1)-\gamma} \\
S=\frac{b^{\gamma(d-1)} r_{+}^{(d-1)(1-\gamma)} \omega_{d-1}}{4} \\
U=\frac{q b^{\gamma(3-d)}}{\Pi r_{+}{ }^{\Pi}} .
\end{gathered}
$$

Using Eq.(8) and relation $V=\int 4 S d r_{+}$, one obtains the volume as

$$
V=\frac{\left(1+\alpha^{2}\right) b^{(d-1) \gamma} \omega_{d-1}}{d+\alpha^{2}} r_{+}^{\left(d+\alpha^{2}\right) /\left(1+\alpha^{2}\right)}
$$

In the extended phase space, the mass of black hole is considered as enthalpy [9] and hence the first law of thermodynamics and Smarr formula take the form

$$
\begin{aligned}
d M & =T d S+U d Q+V d P \\
M & =\frac{(d-1)(1-\gamma)}{\Pi} T S+U Q+\frac{(4 \gamma-2)}{\Pi} V P
\end{aligned}
$$

in which $P$ is the thermodynamic pressure, given by

$$
P=-\frac{\left(d+\alpha^{2}\right) b^{2 \gamma}}{8 \pi\left(d-\alpha^{2}\right) r_{+}^{2 \gamma}} \Lambda .
$$

In the absence of dilaton $(\alpha=0)$, the above pressure becomes $P=-\Lambda / 8 \pi$, which is the pressure of the ReissnerNordstrum-AdS (RN-AdS) black hole [8].

\section{INSTABILITY AND PHASE TRANSITION IN DILATON BLACK HOLE}

The sign of the response functions must be positive for local stability of a thermodynamic system [28]. Since we consider an extended phase space, it is important to study the behavior of the isothermal compressibility

$$
\kappa_{T}=-\left.\frac{1}{V} \frac{\partial V}{\partial P}\right|_{T}
$$




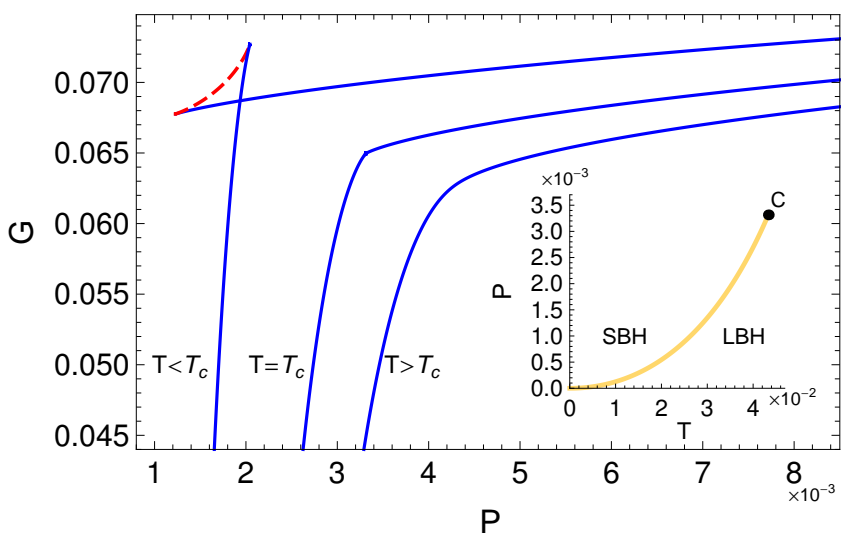

FIG. 1: Gibbs free energy as a function of pressure for $\alpha=0$, $q=1$ and $d=3$ and different values of temperature. Below the critical temperature $\left(T_{c}\right)$, it shows multi-valued behavior indicating a first order phase transition $(\mathrm{SBH} / \mathrm{LBH})$. The positive (negative) sign of $\kappa_{T}$ is identified by blue solid (dashed red) line. Note that various curves are shifted for clarity. The corresponding phase diagram $(P-T)$ is shown in the inset.

Negative sign of $\kappa_{T}$ indicates local thermodynamic instability and therefore a phase transition in the system. To see the exact behavior of thermodynamic system with regard to local instability, we need to calculate the Gibbs free energy $G=G(T, P)$, which is obtained as

$$
\begin{aligned}
G= & M-T S=\left\{\frac{(d-2)\left(1+\alpha^{2}\right) b^{\gamma(n-3)}}{16 \pi\left(\alpha^{2}+d-2\right) r_{+}^{(n-3)(\gamma-1)-1}}\right. \\
& +\frac{P\left(\alpha^{4}-1\right) b^{\gamma(d-1)}}{(d-1)\left(d+\alpha^{2}\right) r_{+}^{d(\gamma-1)-\gamma}} \\
& \left.+\frac{q^{2}\left(2 d-3+\alpha^{2}\right)\left(\alpha^{2}+1\right) b^{\gamma(3-d)}}{8 \pi\left(d-2+\alpha^{2}\right)(d-1) r_{+}^{(n-3)(1-\gamma)+1}}\right\} \omega_{n-1},
\end{aligned}
$$

Let us first consider the Gibbs free energy in the absence of dilaton $(\alpha=0)$, depicted in Fig. 1 for $d=3$. According to Fig.(1), the Gibbs energy is single-valued and increases monotonically with the increasing pressure for $T>T_{c}$ and is locally stable $\left(\kappa_{T}>0\right)$ everywhere as indicated by the solid blue line. However, for $T<T_{c}$, it becomes multi-valued with negative $\kappa_{T}$ shown by dashed red line. Since the minimum value of $G$ is chosen by the system at equilibrium, this indicates a first order phase transition which occurs between small black hole $(\mathrm{SBH})$ and large black hole $(\mathrm{LBH})$ where the slope of $G$ is discontinuous at the transition point. At $T=T_{c}$, a second-order phase transition occurs between SBH and LBH where $G$ is single-valued and continuous, but is nonanalytic. The corresponding phase $(P-T)$ diagram for RN-AdS is illustrated in the inset of Fig.1. SBH is distinguished from LBH by a transition line with a critical point at the end of the transition curve. Note that this transition curve looks analogous to the Van der Waals $P-T$ diagram. The qualitative behavior for higher di-

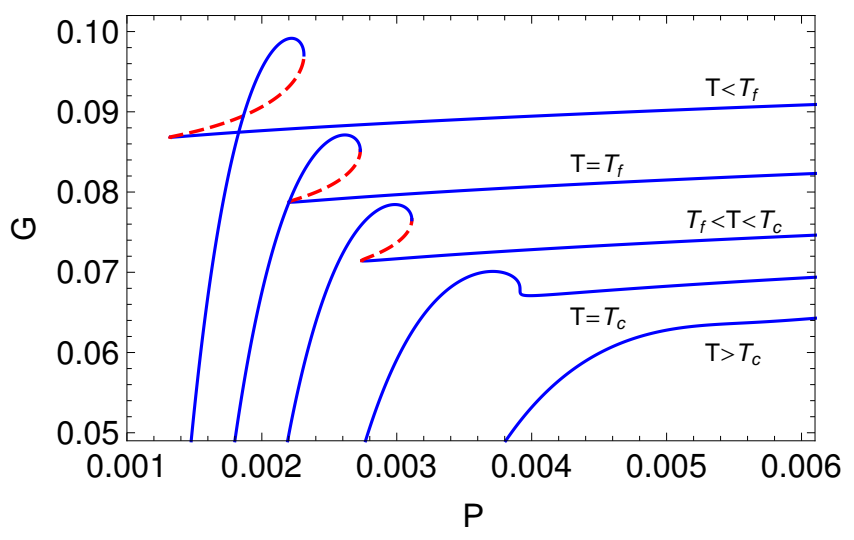

FIG. 2: Gibbs free energy as a function of pressure for different values of temperature and $\alpha=0.4, b=1, q=1$ and $d=3$. The positive (negative) sign of $\kappa_{T}$ is identified by blue solid (dashed red) line. For $T \leq T_{c}, G(P)$ develops non-analytic behavior in the different ways, at $T=T_{c}$, for $T_{\mathrm{f}}<T<T_{c}$ and finally for $T<T_{\mathrm{f}}$. Note that various curves are shifted for clarity.

mensional black holes is the same as $d=3$.

Now, we turn to examine the effects of the dilaton field parameter $(\alpha \neq 0)$ on the phase transition of black holes in extended phase space. For this purpose, we plot the Gibbs free energy as a function of pressure for $\alpha=0.4$ and $d=3$ in Fig. 2. One can see that for $T \geq T_{c}$, the behavior is similar to the previous case $(\alpha=0)$, namely the Gibbs free energy is single-valued and increases monotonically with increasing the pressure. Here $T_{c}$ is the critical point where a second order phase transition occurs. In the range of temperature $T \leq T_{\mathrm{f}}$, where the loop is formed at $T=T_{\mathrm{f}}$, a first order phase transition occurs similarly to the Van der Waals fluid system. However, for the temperature range $T_{\mathrm{f}}<T<T_{c}$ an interesting phenomenon occurs where a finite jump in $G$ leads to a zeroth-order phase transition. Such zeroth-order phase transition has previously been considered in the theory of superfluidity and superconductivity [20], and more recently has been reported as a part of reentrant phase transition in black holes[21-25].

To be more specific, let us consider the case of $T \approx$ $0.064 \in\left[T_{\mathrm{f}}, T_{c}\right]$ in Fig. 3. Decreasing the pressure of the system (despite its multi-valuedness) follows a locally stable regime $\left(\kappa_{T}>0\right)$ by choosing the lower $G$ value. However, at $P=P_{0}$ a jump to a higher (stable) value of $G$ is required thus leading to a discontinuous $G$ and a zeroth order phase transition. Note that this type of phase transitions is absent in normal liquid-gas transitions such as the Van der Waals fluid.

The phase diagram of charged dilaton black hole is shown in Fig. 4. A Van der Waals like first order phase transition occurs for $(T, P)<\left(T_{\mathrm{f}}, T_{\mathrm{f}}\right)$. This is followed by zeroth order phase transition curve with finite jump in $G$ for the range $\left(T_{\mathrm{f}}, T_{\mathrm{f}}\right)<(T, P)<\left(T_{c}, T_{c}\right)$. Interestingly, the zeroth-order transition curve terminates at the critical point $\left(T_{c}, T_{c}\right)$. 


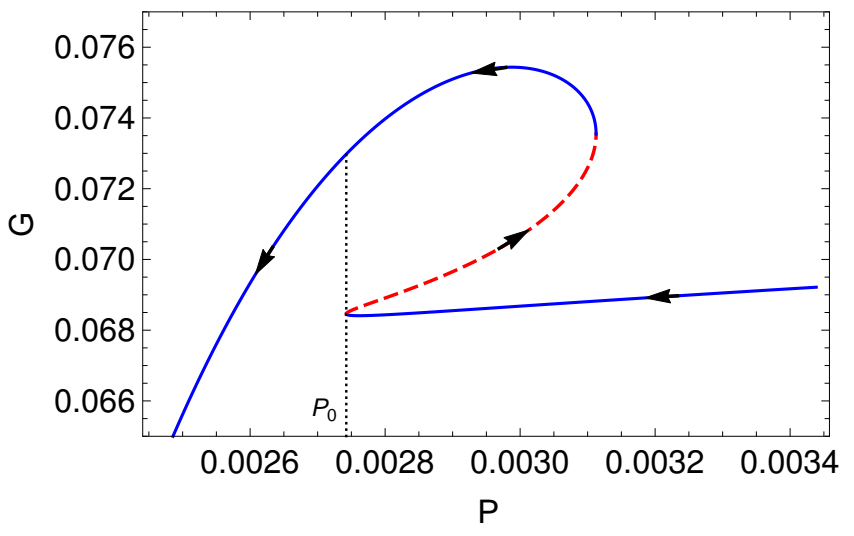

FIG. 3: A close-up of $T \approx 0.064 \in\left[T_{\mathrm{f}}, T_{c}\right]$ in Fig. 2 displays a zeroth order phase transition which is accompanied by a finite jump in $G$ at $P_{0} \approx 0.00274$. The positive (negative) sign of $\kappa_{T}$ is identified by blue solid (dashed red) line and the arrows show the direction of the increasing $r_{+}$.

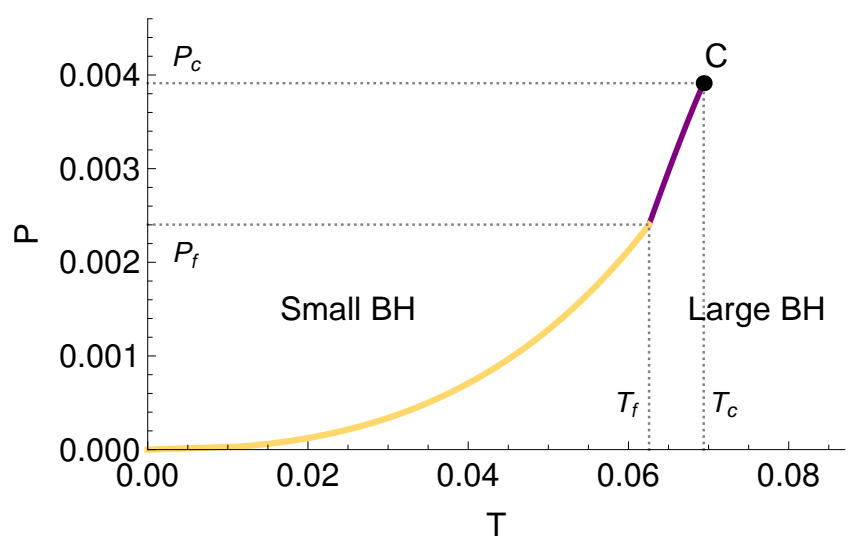

FIG. 4: Phase diagram corresponding to Fig. 2 for transition between $\mathrm{SBH}$ and $\mathrm{LBH}$. Three different transition are identified: first order (gold curve), zeroth order (purple curve) and a second order critical point at $\left(T_{c}, P_{c}\right)$.

\section{EQUATION OF STATE}

The equation of state $P=P\left(T, r_{+}\right)$(for fixed $\left.q\right)$ can be obtained by using Eq.(7) as

$$
\begin{aligned}
P= & \frac{\left(d+\alpha^{2}\right)(d-1) T}{4\left(d-\alpha^{2}\right)\left(1+\alpha^{2}\right) r_{+}}+\frac{(d-2)(d-1)\left(d+\alpha^{2}\right) b^{-2 \gamma}}{16 \pi\left(\alpha^{2}-1\right)\left(d-\alpha^{2}\right) r_{+}^{2-2 \gamma}} \\
& +\frac{\left(d+\alpha^{2}\right) q^{2} b^{-2 \gamma(d-2)}}{8 \pi\left(d-\alpha^{2}\right) r_{+}^{1-(2 d-3)(\gamma-1)+\gamma}}
\end{aligned}
$$

The $P-r_{+}$isothermal diagrams for $\alpha=0.4$ and $d=3$ are shown in Fig. 5. The critical point is essentially an inflection point where $\left(\partial P / \partial r_{+}=0\right.$ and $\left.\partial^{2} P / \partial r_{+}^{2}=0\right)$,

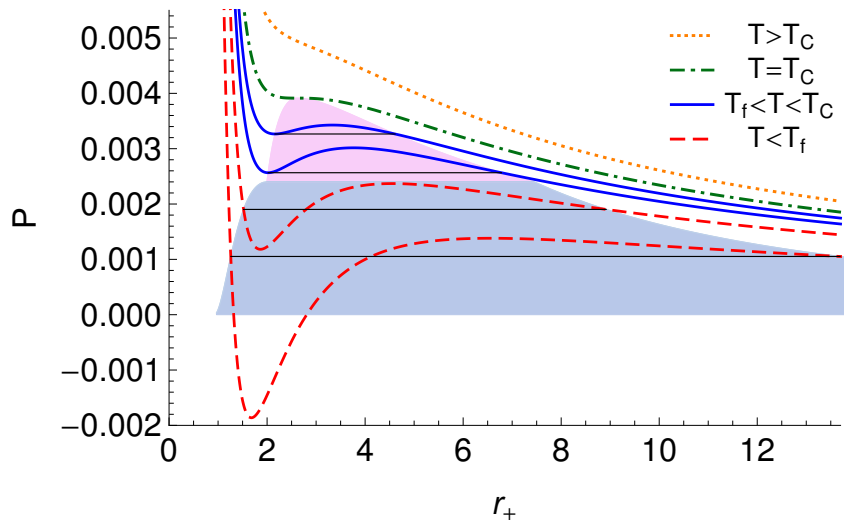

FIG. 5: $P-r_{+}$diagram of charged dilaton black holes for various temperatures and $\alpha=0.4, b=1, q=1$ and $d=3$. The regions of zeroth and first order phase transition are characterized by different colors. The isobars (black thin line) remedy the unphysical locally and globally unstable regimes, see Fig. 2.

and can be obtained as [16]

$$
\begin{aligned}
P_{c}= & {\left[\frac{(d-1)(d-2)}{2\left(2 d-3+\alpha^{2}\right)\left(d-1+\alpha^{2}\right)}\right]^{[\gamma-(2 d-3)(\gamma-1)+1] / 2 \Pi} } \\
& \times\left[\frac{\left(d+\alpha^{2}\right)\left(2 d-3+\alpha^{2}\right)\left(d-2+\alpha^{2}\right)}{\left.8 \pi\left(1+\alpha^{2}\right)\left(d-\alpha^{2}\right) b^{2 \gamma / \Pi} q^{2(1-\gamma) / \Pi}\right],}\right. \\
T_{c}= & {\left[\frac{(d-1)}{2 q^{2}\left(d-1+\alpha^{2}\right)}\right]^{(1-2 \gamma) / 2 \Pi}\left(\frac{\left(\alpha^{2}+d-2\right)}{\pi\left(1-\alpha^{2}\right) b^{\gamma(n-1) / \Pi}}\right) } \\
& \times\left[\frac{\left(2 d-3+\alpha^{2}\right)}{(d-2)}\right]^{[(2 d-3)(\gamma-1)-\gamma] / 2 \Pi}, \\
r_{+c}= & {\left[\frac{2 q^{2}\left(d-1+\alpha^{2}\right)\left(2 d-3+\alpha^{2}\right) b^{6 \gamma-2 d \gamma}}{(d-2)(d-1)}\right]^{1 / 2 \Pi} }
\end{aligned}
$$

According to Eq. (18), critical temperature has positive values if dilaton parameter is restricted to $\alpha<1$. One can calculate the critical exponents $\alpha^{\prime}=0, \beta^{\prime}=1 / 2$, $\delta^{\prime}=3$ and $\gamma^{\prime}=1$ associated with second order transition which are mean-field values such as the Van der Waals fluid system [16].

For $T<T_{c}$, we observe local instability $\left(\kappa_{T}<0\right)$ and negative pressure in some range of quantities for the system. Such an unphysical behavior is remedied by choosing the globally stable Gibbs free energy as illustrated in Fig. 2. For $T<T_{\mathrm{f}}$ where a first order phase transition occurs, a globally unphysical part is replaced by the isobar line for which $G_{\mathrm{SBH}}=G_{\mathrm{LBH}}$. Following the behavior of Gibbs free energy, we observe that the modified isobar line for the zeroth order phase transition starts at the point of local minimum of isotherm for range $T_{\mathrm{f}}<T<T_{c}$. One can see from Fig. 5 that $\kappa_{T}$ diverges $\left(\partial P / \partial r_{+}=0\right)$ in the case of zeroth order phase transition. Similarly, one can plot the entropy $(S)$ versus temperature $(T)$ as well, and will subsequently see that the heat capacity at the constant pressure diverges in zeroth order phase transition. 


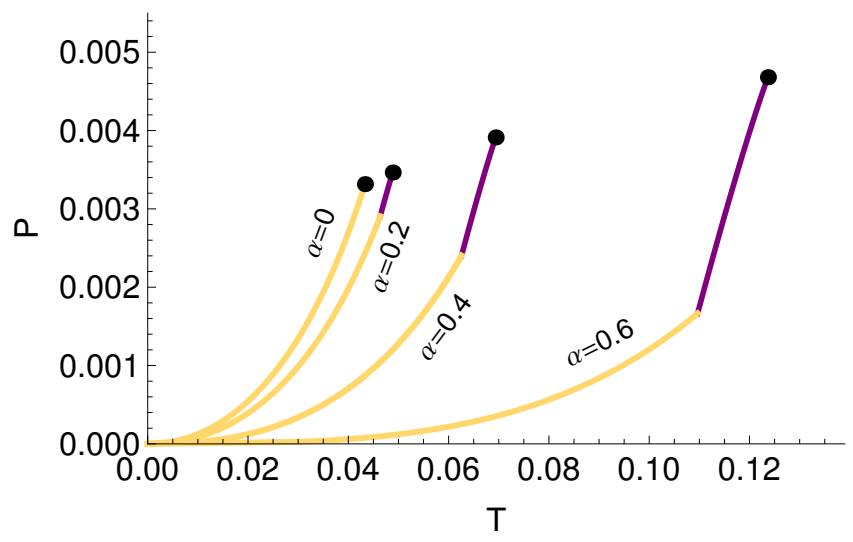

FIG. 6: SBH/LBH phase diagram for various values of dilaton parameter $\alpha$ and $b=1, q=1$ and $d=3$. Increasing $\alpha$ leads to a larger portion of the transition curve belonging to zeroth order phase transition. The critical points are highlighted by a black solid circle.

Fig. 6 shows SBH/LBH phase diagram for various values of dilaton parameter $\alpha$. As is seen from the figure increasing $\alpha$ from zero (RN-AdS BH) will lead to creation and elongation of the zeroth order phase transition as the critical point moves higher and the first order transition curve (purple) bends lower and further in the $P-T$ space. Finally, it is worth mentioning that similar qualitative behavior for the higher dimensional $(d>3)$ charged dilaton black holes can be observed.

\section{SUMMARY}

In this paper, we have investigated the thermodynamic phase behaviour of charged dilaton black holes in the presence of Liouville-type dilaton potentials. Due to the presence of the dilaton field, these solutions are neither asymptotically flat nor (A)dS [26]. We have disclosed the effects of the dilaton field on the phase transition properties of charged black holes in an extended phase space. In addition to the usual small/large black holes transition (first and second order) [7, 8], we observed a zeroth order phase transition between small/large black hole in which isothermal compressibility and heat capacity at the constant pressure diverge. Besides, the Gibbs free energy has a finite jump, at the point where a zeroth order phase transition occurs. We also obtained that a zeroth order phase transition emerges in a longer portion of the transition line by increasing a coupling constant of dilaton field. It is worth noting that a coupling constant is restricted to $\alpha<1$, for which the critical temperature is positive. Finally, we showed a set of critical exponents which are the same with Van der Waals fluid system.

\section{Acknowledgments}

Shiraz University research council is kindly acknowledged. The work of AS has been supported financially by Research Institute for Astronomy \& Astrophysics of Maragha (RIAAM), Iran.
[1] J.D. Bekenstein, Phys. Rev. D 7, 2333 (1973); J.D. Bekenstein, Phys. Rev. D 9, 3292 (1974).

[2] S.W. Hawking, Commun. Math. Phys. 43, 199 (1975); S.W. Hawking, Phys. Rev. D 13, 191 (1976).

[3] S. Hawking and D. N. Page, Commun.Math.Phys. 87, 577 (1983).

[4] A. Chamblin, R. Emparan, C. Johnson, and R. Myers, Phys.Rev. D 60, 064018 (1999); A. Chamblin, R. Emparan, C. Johnson, and R. Myers, Phys. Rev. D 60, 104026 (1999).

[5] H. B. Callen, 2nd ed. (John Wiley \& Sons, New York, 1985).

[6] D. C. Johnston, arXiv:1402.1205.

[7] B. P. Dolan, Class. Quant. Grav. 28,125020 (2011); B. P. Dolan, Class. Quant. Grav. 28, 235017 (2011).

[8] D. Kubiznak, R. B. Maan, JHEP 07, 033 (2012).

[9] D, Kastor, S, Ray, and J, Traschen, Class. Quant. Grav. 26, 195011 (2009).

[10] B. P. Dolan, Phys.Rev. D 84, 127503 (2011); B. P. Dolan, Fortsch. Phys. 62, 892 (2014).

[11] M. Cvetic, G. W. Gibbons, D. Kubiznak, and C. N. Pope, Phys. Rev. D 84, 024037 (2011).

[12] M. Urana, A. Tomimatsu, and H. Saida, Class. Quant. Grav. 26, 105010 (2009).

[13] S.-W. Wei and Y.-X. Liu, Phys. Rev. D 87, 044014 (2013).

[14] De. Zou, Y.i Liu, B. Wang, Phys. Rev. D 90, 044063
(2014).

[15] S. H. Hendi, M. H. Vahidinia, Phys. Rev. D 88, 084045 (2013); S. H. Hendi, S. Panahiyan, B. Eslam Panah, Int. J. Mod. Phys. D Vol. 25, No. 1, 1650010 (2016).

[16] M. H. Dehghani, S. Kamrani, A. Sheykhi, Phys. Rev. D. 90 104020,(2014).

[17] M. H. Dehghani, A. Sheykhi and Z. Dayyani, Phys. Rev. D 93, 024022 (2016).

[18] Z. Dayyani, A. Sheykhi and M. H. Dehghani, Phys Rev D 95, 084004 (2017).

[19] A. Dehyadegari, A. Sheykhi and A. Montakhab, Phys. Lett. B 768, 235 (2017).

[20] V. P. Maslov, Math Notes 76, 697 (2004).

[21] S. Gunasekaran, R. B. Mann and D. Kubiznak, JHEP 11, 110 (2012).

[22] N. Altamirano, D. Kubiznak, and R. B. Mann, Phys. Rev. D. 88, 101520 (2013); N. Altamirano, D. Kubiznak, R. B. Mann and Z. Sherkatghanad, Class. Quant. Grav. 31, 042001 (2014); S. W. Wei, P. Cheng and Y. X. Liu, Phys. Rev. D 93, 084015 (2016).

[23] A. M. Frassino, D. Kubiznak, R. B. Mann and F. Simovic, JHEP 1409, 080 (2014); De. Zou, Y. Lio, B. Wang, Phys. Rev. D. 90, 044063 (2014) ;R. A. Hennigar, W. G. Brenna and R. B. Mann, JHEP 1507, 077 (2015); M. B. Jahani Poshteh, B. Mirza, Z. Sherkatghanad, Phys. Rev. D 88, 024005 (2013).

[24] R. A. Hennigar and R. B. Mann, Entropy 17, 8056 
(2015)

[25] N. Altamirano, D. Kubiznak, R. B. Mann and Z. Sherkatghanad, Galaxies 2, 89 (2014); D. Kubiznak and F. Simovic, Class. Quant. Grav. 33, 245001 (2016).

[26] A. Sheykhi, Phys. Rev. D 76, 124025 (2007).
[27] K. C. K. Chan, J. H. Horne, and R. B. Mann, Nucl. Phys. B447, 441 (1995).

[28] H. B. Callen, 2nd ed. (John Wiley \& Sons, New York, 1985). 